\title{
Extended ensemble molecular dynamics method for constant strain rate uniaxial deformation of polymer systems
}

\author{
Liu Yang, David J. Srolovitz, and Albert F. Yee \\ Department of Materials Science and Engineering, University of Michigan, Ann Arbor, \\ Michigan 48109-2136
}

(Received 4 December 1996; accepted 27 May 1997)

\begin{abstract}
We describe a novel molecular dynamics (MD) method to simulate the uniaxial deformation of an amorphous polymer. This method is based on a rigorously defined statistical mechanics ensemble appropriate for describing an isothermal, displacement controlled, uniaxial stress mechanical test. The total number of particles is fixed and the normal stresses in the direction normal to the applied strain are constant, i.e., an $N T L_{x} \sigma_{y y} \sigma_{z z}$ ensemble. By using the Lagrangian of the extended system (i.e., including additional variables corresponding to the temperature and cross-sectional area fluctuations), we derive a set of equations of motion for the atomic coordinates and the additional variables appropriate to this ensemble. In order to avoid the short MD time step appropriate for the stiff covalent bonds along the polymer chains, we introduce bond length constraints. This is achieved using a variation of the commonly used SHAKE [J. P. Ryckaert, G. Ciccotti, and H. J. C. Berendsen, J. Comp. Phys. 23, 327 (1977)] algorithm. A numerical method for integrating the equations of motion with constraints via a modification of the velocity Verlet [W. C. Swope, H. C. Andersen, P. H. Berens, and K. R. Wilson, J. Chem. Phys. 76, 637 (1982)] algorithm is presented. We apply this new algorithm to the constant strain rate deformation of an amorphous polyethylene in a model containing several distinct polymer chains. To our knowledge, this is the first time that bond length constraints were applied to a macromolecular system together with an extended ensemble in which the simulation cell shape is allowed to fluctuate. (C) 1997 American Institute of Physics. [S0021-9606(97)50133-4]
\end{abstract}

\section{INTRODUCTION}

The mechanical properties of polymers play an important role in effectively all polymer applications. Therefore, these properties have received considerable attention of the polymer research community. The macroscopic mechanical properties of amorphous polymers are intimately related to their microscopic molecular structure and the types of molecular motions that occur upon application of a stress. Yield behavior is one of the most important attributes of an amorphous polymers. How a polymer plastically deforms controls such important properties as ductility, toughness and impact resistance.

The deformation behavior of amorphous polymers has been studied experimentally, theoretically, and via computer simulation. Experimental investigations have the advantage that they provide macroscopic and/or microscopic data on specific, real materials. However, in experimental work, it is difficult to separate the effects arising from different origins and to systematically vary specific structural properties without inadvertently modifying other properties. Unlike in experimental work, theoretical approaches allow us to change individual parameters without affecting others. Because of the inherent complexity of the yielding phenomena in polymers, theoretical analyses are often reduced to describing plastic behavior in terms of composite parameters that are empirically chosen to provide the best fit with experimental observations. Molecular scale simulations have the advantage over analytical theory and experiment in that the physical behavior can be traced back to their specific structural origin. Simulations also have the advantage of being able to systematically vary specific structural features (e.g., bond torsional stiffness) without modifying others. Unfortunately, within the capabilities of present-day computing, molecular simulations are limited in terms of spatial and temporal scales that can be investigated. Another limitation of molecular simulations is their dependence on (usually) empirical descriptions of atomic interactions. Given these limitation, applications and interpretation of molecular scale simulations have to be carefully chosen to avoid artifacts.

While there have been many computer simulation studies of deformation, they have focused predominantly on the deformation of atomic, rather than macromolecular, systems. Relatively few computer simulation studies of the deformation of solid amorphous polymers have been performed. This is due, in part, to the added complexity associated with the connectivity of polymer chains. This factor is, however, very important because the difference between the mechanical behavior of macromolecular systems and atomic systems is primarily attributable to this very feature. Argon, Mott, Hutnik, and Suter ${ }^{1-3}$ have used a computer simulation procedure, based upon energy minimization, to study the yielding behavior of several polymer systems. These studies were the first to make the important connection between specific features of the stress-strain curve and molecular scale phenomena. Because their simulations were based upon the energy minimization method, no information could be directly extracted regarding the role played by temperature or strain rate in the deformation of these amorphous polymers. The simu- 
lation approach employed by Argon et al. ${ }^{1-3}$ was limited to fixed Poisson's ratio deformation $(\nu=0.5)$, such that the overall density was fixed. This is, potentially, a major limitation, since density changes do occur during deformation and such changes are often invoked to explain part of the deformation behavior of polymers.

The molecular dynamics (MD) simulation method has several advantages over the energy minimization approach in studying such dynamical phenomena as deformation. For example, MD can be used to predict the detailed dynamics associated with deformation events and to examine, directly, temperature and strain rate effects. Brown, Clarke, and co-workers ${ }^{4,5}$ were the first to apply the MD method to study the yielding behavior of a macromolecular system. Their study employed a "loose-coupling" MD procedure which is not based on a rigorously defined statistical ensemble. Further, their use of a fictitious external stress resulted in deformation that was neither constant strain or stress rate. This makes the significance of their results difficult to evaluate. In order to overcome these difficulties, we have developed a new molecular dynamics simulations procedure for modeling the deformation behavior of amorphous polymers that is rigorously based on a prescribed statistical ensemble and allows for constant strain rate simulations that closely reproduce the deformation history and behavior that occur in uniaxial tests commonly performed in the laboratory.

Deformation experiments on amorphous polymers are typically performed using a testing machine in which the cross-head displacements are specified in order to maintain a prescribed strain rate in one direction (such displacement controlled experiments are much easier to perform than stress controlled experiments). The normal stresses in the two directions perpendicular to the tensile axis are zero (actually atmospheric pressure). The advantage of strain controlled tests over stress controlled tests is that the former permits the investigation of post-yield stress drop deformation. While such uniaxial tests are not explicitly isothermal, typical variations in temperature during a tensile test at typical testing rates do not exceed $1 \mathrm{~K}$. Therefore, the new, extended ensemble molecular dynamics method developed here correspond to a constant, uniaxial strain rate test in which the transverse normal stresses are zero, the temperature is constant, and the stress is purely uniaxial. While the procedure developed here was designed to model the constant strain rate, uniaxial tensile test of an amorphous polymer, it is sufficiently general to simulate any type of strain controlled uniaxial test (tension or compression) of any type of material.

In the next section of this paper, we describe the conceptual framework of the new, extended ensemble (isothermal, constant uniaxial strain rate) MD method. A detailed description of this approach, as it applies to atomic systems is the subject of Sec. III. Exactly the same procedure could be used to simulate polymeric systems. However, since the in-chain covalent bonds are much stiffer than the bending, torsional and van der Waals portions of the interatomic potential, these stiff bonds force the use of very short simulation time steps. In order to perform longer time scale simulations, it is advantageous to fix the bond length. Ryckaert and co-workers $^{6-8}$ devised an approach, known as the SHAKE algorithm to handle this constraint. In Sec. IV, we combine our generalized extended ensemble MD method to account for bond length constraints using a variation of the SHAKE algorithm. Next, we describe the numerical procedures and several important practical details used to successfully integrate the equations of motions derived in Sec. IV. Finally, we present the results from our simulations of the deformation of a model, amorphous polyethylene performed using our new, extended ensemble MD method.

\section{II. $N T L_{x} \sigma_{y y} \sigma_{z z}$ EXTENDED ENSEMBLE MD}

MD is a simulation approach in which the equations of motion of each particle are numerically integrated forward in time. Given initial positions and momenta of all particles and a description of the interaction of the particles, MD can be used to predict the trajectories of all of the particles. The macroscopic behavior of the system is determined by performing (time) averages of the desired properties over all of the trajectories. If the ergodic hypothesis holds, the trajectory average of a property is equivalent to the corresponding ensemble average. Therefore, in order to determine the appropriate macroscopic properties, it is essential that the ensemble used to generate the particle trajectories is suitably chosen to match the appropriate macroscopic boundary conditions. In traditional molecular dynamics, the classical Newtonian equations of motion are solved for each particle producing trajectories corresponding to the microcannonical ensemble. This is called the $N V E$ ensemble, since the number of particles $(N)$, total volume $(V)$, and energy $(E)$ are conserved.

The extended ensemble concept, first proposed by Andersen, ${ }^{9}$ greatly enhances the power and flexibility of MD. Andersen used this method to perform simulations in which the pressure and enthalpy were conserved (i.e., $N P H$ ) rather than the volume and energy $(N V E)$. This was done by introducing the system volume as a dynamical variable. This volume was determined in terms of the deviation of the internal pressure of the system from the specified external pressure. In this extended ensemble method, a set of "extended" or "virtual" variables (in this case, the scaled particle coordinates and the volume) and a properly modified Lagrangian are introduced. The Hamiltonian and equations of motion of the "extended" variables are derived from the Lagrangian. By defining the direct relationships between the positions and momenta of the "original system" with the "extended" variables, every state of the extended system corresponds to a unique point in the phase space of the original system. The time scale associated with the volume fluctuation is determined by the choice of a constant in the modified Lagrangian, which can be interpreted as the inertia of a piston. The time average of any property calculated from the trajectory generated by the equations of motion of the extended system is equal to the ensemble average of this property for an isoenthalpic-isobaric ensemble in which the pressure is the specified value. ${ }^{9}$ Parrinello and Rahman ${ }^{10}$ 


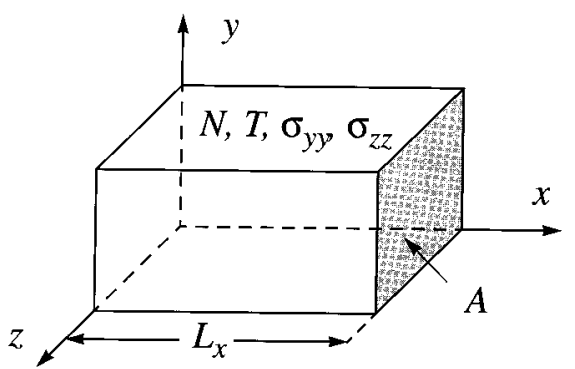

FIG. 1. The simulation cell geometry and coordinate system used to model the $N T L_{x} \sigma_{y y} \sigma_{z z}$ ensemble.

generalized this method to the case of constant stress by introducing the lengths and angles describing the box containing the system as additional variables to be set by the external stress (tensor). Nosé ${ }^{11}$ proposed yet another variation of this procedure in order to keep the temperature constant (i.e., the $N V T$ ensemble). To this end, he introduced a new variable $s$ to scale the MD time step. $s$ is related to the specific heat of the thermal reservoir in contact with the system.

In the present paper, we focus on the ensemble appropriate for modeling a uniaxial tension test. The required ensemble will be isothermal, which corresponds to a prescribed strain in the $x$-direction and fixed normal stresses in the $y$ and $z$-directions. To this end, we first generalize the extended ensemble method to describe an isothermal orthorhombic system in which the dimension of the simulation cell in the $x$-direction and the normal stresses in the other two directions are fixed. This ensemble is illustrated in Fig. 1. We refer to this as the $N T L_{x} \sigma_{y y} \sigma_{z z}$ ensemble, the Lagrangian and equations of motion for which are derived in the following section. Constant strain rate simulations can be performed by appropriately choosing the rate at which the length of the box is changed.

While traditional ( $N V E)$ MD simulations are performed at fixed volume and energy, thereby leaving the stress and temperature uncontrolled, in the $N T L_{x} \sigma_{y y} \sigma_{z z}$ ensemble the length of the box, the temperature and the transverse normal stresses are fixed while the longitudinal stress $\left(\sigma_{x x}\right)$, the energy and the cross sectional area $A$ fluctuate. Therefore, this ensemble is like an NVT ensemble in the $x$-direction and, at the same time, like an NPT ensemble in the $y$ and $z$-directions. A natural choice is to break the symmetry of the $x, y$ and $z$-directions and consider them differently. Since we specify the length of the simulation cell in the $x$-direction and the normal stress in the $y$ and $z$-directions (in response to the external pressure), we introduce the cross section area of the simulation cell in the $y z$-plane $A$ as a dynamic variable. $A$ will fluctuate as necessary in order to keep the normal stresses in $y$ and $z$-directions fixed. In principle, we should employ two dynamic variables, corresponding to the dimensions of the simulation cell in the $y$ and $z$-directions; however, since these two directions are equivalent in an amorphous polymer, we employ only the single dynamic variable $A$. As in the $N V T$ extended ensemble method of Nosé, we introduce the dynamic variable $s$ to control the temperature. Two constants $w_{A}$ and $w_{s}$ are introduced into the Lagrangian, and they are associated with the dynamic fluctuations of $A$ and $s$, respectively.

In the next section, we present a detailed description of the "extended variables" and the relationship between the extended variables and the real system variables. In addition, we present the Lagrangian, Hamiltonian, and equations of motion for the extended system. In the Appendix, we extend the proof presented by Nosé, ${ }^{11}$ to demonstrate that the configurations generated by these equations have exactly the same partition function as the $N T L_{x} \sigma_{y y} \sigma_{z z}$ ensemble.

\section{ATOMIC SYSTEMS}

In this section, we focus on the $N T L_{x} \sigma_{y y} \sigma_{z z}$ ensemble of $N$ atomic particles interacting through interatomic potentials which are known analytically. The "extended" variables of this system include $\left\{x_{i}, y_{i}, z_{i}, A, s\right\}$, as discussed above. The atomic positions $\left\{x_{i}, y_{i}, z_{i}\right\}$ are scaled by the overall shape of the simulation cell dimensions

$$
\mathbf{r}_{i}^{\text {real }}=\mathbf{h}\left(\begin{array}{c}
x_{i} \\
y_{i} \\
z_{i}
\end{array}\right)=\left(\begin{array}{ccc}
L_{x} & 0 & 0 \\
0 & \sqrt{A} & 0 \\
0 & 0 & \sqrt{A}
\end{array}\right)\left(\begin{array}{c}
x_{i} \\
y_{i} \\
z_{i}
\end{array}\right),
$$

where $\mathbf{h}$ is the matrix describing the orthorhombic simulation cell and is defined within the equation. In this scaling, the coordinates $x_{i}, y_{i}, z_{i} \in[0,1)$ and the dimensions of the simulation cell in the $x, y$, and $z$-directions are $L_{x}, \sqrt{A}$, and $\sqrt{A}$. Note, that while $L_{x}$ is specified in the ensemble, $A$ will fluctuate in time. In this ensemble, if the coordinate axes are chosen to lie parallel to the edges of the orthorhombic unit cell, all of the off-diagonal terms in the stress tensor $\sigma$ are zero (by the symmetry of the loading and the orthorhombic unit cell), $\sigma_{y y}$ and $\sigma_{z z}$ are constrained to be equal to the external pressure $P_{\text {ext }}$, and $\sigma_{x x}$ fluctuates (because $L_{x}$ is fixed).

The dynamics of the system will be controlled by the Lagrangian

$$
\begin{aligned}
L= & \sum_{i}^{N} \frac{m_{i}}{2} s^{2}\left(L_{x}^{2} \stackrel{*}{x}_{i}^{2}+A{\stackrel{*}{y_{i}}}^{2}+A{\stackrel{*}{z_{i}}}^{2}\right)-\Phi+\frac{w_{s}}{2} \stackrel{*}{s}^{2} \\
& -(f+1) k_{B} T_{\mathrm{ext}} \ln s+\frac{\stackrel{*}{w} A}{2} \stackrel{*}{A}^{2}-P_{\mathrm{ext}} L_{x} A,
\end{aligned}
$$

which is a function of the "extended" variables $\left\{x_{i}, y_{i}, z_{i}, A, s\right\}$ and of their time derivatives with respect to "virtual" time $\tau$ (as described later in this section). In this function, $m_{i}$ is the mass of particle $i, \Phi$ is the energy of the system determined by summing the interatomic potentials and the asterisks indicate differentiation with respect to the "virtual" time (as opposed to real time, as described below). $s$ is the dynamic variable which is used to maintain the system at constant temperature $T_{\text {ext }}$ and is associated with the scaling of the time step in MD and $w_{s}$ determines the magnitude of the temperature fluctuations, as originally introduced by Nosé. ${ }^{11} f$ is the number of degrees of freedom of 
the system $[f=d(N-1)]$, where $d$ is the dimensionality of space. $w_{A}$ may be viewed as the inertia of the piston which fixes the normal stress in the $y$ and $z$-directions at the external pressure $P_{\text {ext }}$, similar to the approach introduced by Andersen. ${ }^{9}$

The first and the second terms in the Lagrangian are associated with the kinetic energy and the potential energy of the system; the third and the forth terms can be viewed as the kinetic energy and the potential energy associated with dynamical variable $s$; the fifth and sixth terms are associated with the kinetic and the potential energy of the dynamic variable $A$. The momenta of the "extended" variables $\left\{x_{i}, y_{i}, z_{i}, A, s\right\}$ can be derived from this Lagrangian,

$$
\begin{aligned}
& \pi_{x i}=m_{i} s^{2} L_{x}^{2} \stackrel{*}{x}_{i}, \\
& \pi_{y i}=m_{i} s^{2} A \stackrel{*}{y}, \\
& \pi_{z i}=m_{i} s^{2} A \stackrel{*}{z_{i}}, \\
& \pi_{s}=w_{s} s, \\
& \pi_{A}=w_{A} \stackrel{*}{A} .
\end{aligned}
$$

The corresponding Hamiltonian is

$$
\begin{aligned}
H= & \sum_{i}^{N}\left[\frac{1}{2 m_{i} s^{2}}\left(\frac{\pi_{x i}^{2}}{L_{x}^{2}}+\frac{\pi_{y i}^{2}}{A}+\frac{\pi_{z i}^{2}}{A}\right)\right]+\Phi+\frac{\pi_{s}^{2}}{2 w_{s}} \\
& +(f+1) k_{B} T_{\text {ext }} \ln s+\frac{\pi_{A}^{2}}{2 w_{A}}+P_{\text {ext }} L_{x} A .
\end{aligned}
$$

The equations of motion for the "extended" variables, expressed in "virtual"' time, are determined from the Lagrangian,

$$
\begin{aligned}
& \stackrel{* *}{x}_{i}=\frac{f_{x i}}{m_{i} s^{2} L_{x}}-\frac{2 \stackrel{*}{s}}{s} \stackrel{*}{x}, \\
& y_{i}^{* *}=\frac{f_{y i}}{m_{i} s^{2} \sqrt{A}}-\frac{2 \stackrel{*}{s}}{s} \stackrel{*}{y}_{i}-\frac{\stackrel{*}{A}}{A}{ }^{*}{ }_{i}, \\
& z_{i}^{*}=\frac{f_{z i}}{m_{i} s^{2} \sqrt{A}}-\frac{2 \stackrel{*}{s}}{s}{ }^{*} z_{i}-\frac{\stackrel{*}{A}}{A}{ }_{z}^{*}, \\
& \stackrel{* *}{s}=\frac{1}{w_{s} s}\left[\sum_{i} m_{i} s^{2}\left(L_{x}^{2} \stackrel{*}{x}_{i}^{2}+A \stackrel{*}{y}{ }_{i}^{2}+A \stackrel{*}{z_{i}^{2}}\right)\right. \\
& \left.-(f+1) k_{B} T_{\text {ext }}\right] \text {, } \\
& \stackrel{* *}{A}=\frac{L_{x}}{w_{A}}\left[\sum_{i} \frac{m_{i} s^{2}}{2 L_{x}}\left({\stackrel{*}{y_{i}^{2}}}^{2}+\stackrel{*}{z}_{i}^{2}\right)\right. \\
& \left.+\frac{1}{2 \sqrt{A} L_{x}} \sum_{i}\left(f_{y i} y_{i}+f_{z i} z_{i}\right)-P_{\text {ext }}\right],
\end{aligned}
$$

where $f$ is the force on individual atoms derived from gradients of the potential $\Phi$. These equations of motion show that the acceleration of the particle depends on terms related to the temperature control and the box shape, in addition to the forces derived from potentials. The equation of motion for the variable $s$ depends on the difference between the instantaneous kinetic energy of the system and the kinetic energy appropriate for the specified temperature $T_{\text {ext }}$. Similarly, the equation of motion for the variable $A$ depends on the difference between the instantaneous average normal stress in the $y$ and $z$-directions and the external pressure.

The equations of motions described above are for the extended variables or scaled coordinates of the atoms, which are related to the real variables as per Eq. (3.1). The relationship between the "real" time $t$ and the "virtual" time $\tau$ is

$$
d t=d \tau / s .
$$

The unscaled momenta of the real variables are related to the momenta of the extended variables by

$$
\left\{\begin{array}{l}
p_{x i}=\pi_{x i} / s L_{x} \\
p_{y i}=\pi_{y i} / s \sqrt{A} \\
p_{z i}=\pi_{z i} / s \sqrt{A} \\
p_{s}=\pi_{s} \\
p_{A}=\pi_{A} .
\end{array}\right.
$$

The resulting equations of motion in the real variables and real time are found using Eqs. (3.5), (3.1), (3.6), and (3.7),

$$
\begin{aligned}
m_{i} \ddot{r}_{x i}= & f_{x i}-\dot{s} s^{-1} p_{x i} \\
m_{i} \ddot{r}_{y i}= & f_{y i}-\dot{s} s^{-1} p_{y i}+\frac{m_{i} r_{y i}}{2 A}\left(\ddot{A}-\frac{\dot{A}^{2}}{2 A}\right) \\
m_{i} \ddot{r}_{z i}= & f_{z i}-\dot{s} s^{-1} p_{z i}+\frac{m_{i} r_{z i}}{2 A}\left(\ddot{A}-\frac{\dot{A}^{2}}{2 A}\right) \\
w_{s} \ddot{s}= & w_{s} \frac{\dot{s}^{2}}{s}+s\left[\sum_{i=1}^{N} \frac{p_{x i}^{2}+p_{y i}^{2}+p_{z i}^{2}}{m_{i}}-(f+1) k_{B} T\right] \\
w_{A} \ddot{A}= & w_{A} \frac{\dot{s} \dot{A}}{s}+s^{2} L_{x} \\
& \times\left[\frac{1}{2 A L_{x}} \sum_{i=1}^{N}\left(\frac{p_{y i}^{2}+p_{z i}^{2}}{m_{i}}+f_{y i} r_{y i}+f_{z i} r_{z i}\right)-P_{\mathrm{ext}}\right]
\end{aligned}
$$

where $\mathbf{p}_{i}$ is a known function of $\mathbf{r}_{i}, \dot{\mathbf{r}}_{i}, A$, and $\dot{A}$,

$$
\begin{aligned}
& p_{x i}=m_{i} \dot{r}_{x i}, \\
& p_{y i}=m_{i} \dot{r}_{y i}-\frac{m_{i} \dot{A}}{2 A} r_{y i}, \\
& p_{z i}=m_{i} \dot{r}_{z i}-\frac{m_{i} \dot{A}}{2 A} r_{z i} .
\end{aligned}
$$

Using the approach followed by Nosé, ${ }^{11}$ it can be shown that the real phase space trajectory of the system rigorously satisfies the constraints of the $N T L_{x} \sigma_{y y} \sigma_{z z}$ ensemble, as proven in the Appendix.

While it is possible to write and integrate the equations of motion [Eq. (3.5)] directly in terms of the scaled variables and "virtual" time, performing such calculations in terms of the unscaled variables [Eqs. (3.8) and (3.9)] is equivalent. 
However, performing the simulations in real time yields data that are uniformly distributed in time, which makes the performance of time averages more straightforward. Another advantage in performing the simulations using the real (unscaled) variables is associated with the bond length constraints (discussed in the next section), which are most simply written in terms of real atom separations.

In order to perform simulations at a constant, finite engineering strain rate, we slowly increase the $x$-dimension $L_{x}$ of the orthorhombic box. In practice, this is accomplished by increasing $L_{x}$ in finite steps $d L_{x}=\dot{\epsilon} L_{x 0} d t$ at each MD time step, where $d t$ is the time step, $\dot{\epsilon}$ is the strain rate and $L_{x 0}$ is the initial dimension of the sample in the $x$-direction. This approach is valid provided that $d L_{x} / d t$ $=\dot{\epsilon} L_{x 0}$ is much smaller than the speed of sound in the material.

\section{CONSTRAINED MOLECULAR SYSTEMS}

For a polymer system, the intrachain covalent bond is much stiffer than the other types of potentials describing the interactions (i.e., bending, torsional, and van der Waals). Since the time step in a MD simulation must be small compared with the fundamental vibrational period $\Delta t$ $=2 \pi \sqrt{m / k}$ ( $k$ is the bond stiffness and $m$ is the particle mass), large stiffness necessarily implies small MD time steps. Since there is a large disparity between the stiffnesses of the intrachain covalent bond and those of the other types of potentials, it is computationally advantageous to freeze the intrachain covalent bonds at their equilibrium lengths. This is reasonable since the large stiffness of the covalent bonds suggests that the vibrations of these bonds will be of very small amplitude. Furthermore, since the intrachain covalent bonds are much stiffer than the other potentials describing the atomic interactions (i.e., bending, torsional, and van der Waals), constraining these bonds to a fixed length will have very little influence on the deformation behavior. Therefore, introducing bond length constraints should not introduce any serious artifacts into MD simulations of realistic polymer systems, while greatly accelerating the MD simulations.

Ryckaert, Ciccotti, and Berendsen ${ }^{6}$ devised an efficient algorithm using Cartesian coordinates, known as SHAKE, to fulfill the bond length constraint at each MD step. This algorithm does not significantly increase the numerical errors associated with integrating the equations of motion. The incorporation of the SHAKE algorithm into the numerical framework of the Verlet algorithm ${ }^{12}$ (commonly used to integrate the equations of motion in MD) is straightforward.

The SHAKE algorithm was originally developed for traditional $N V E$ systems and its application to other statistical mechanics ensembles (e.g., $N P H, N P T$ ) requires additional theoretical development. By decoupling the internal geometry constraints from the space scaling implicit in the extended system method, Ryckaert, Ciccotti, and Ferrario ${ }^{7,8}$ made the extension of the SHAKE algorithm to other statistical mechanics ensembles possible. In their approach, the Lagrangian of the system is recast in terms of the molecular coordinates of the centers of mass and the relative atomic coordinates. The time scaling, related to temperature control in an NPT ensemble, is applied to all of the degrees of freedom. At the same time, the space scaling is only applied to the centers of mass of each molecule without affecting the relative coordinates of the atoms belonging to the same molecule. We will follow this same general approach to extend the SHAKE algorithm to account for the extended ensemble method described in Sec. IV for the $N T L_{x} \sigma_{y y} \sigma_{z z}$ ensemble.

We can rewrite the Lagrangian for the $N T L_{x} \sigma_{y y} \sigma_{z z}$ ensemble [Eq. (3.2)] in terms of center of mass variables for the individual polymer chains; $\xi_{i \alpha}, \rho_{\alpha}$, where $\alpha$ labels individual chains, $\rho_{\alpha}$ is the scaled position of the center of mass of chain $\alpha$, and $\xi_{i \alpha}$ is the position of atom $i$ in chain $\alpha$ measured relative to the chain center of mass. These new variables are "extended variables" in the sense that they are in the scaled coordinate system and their time derivatives are always with respect to a "virtual" time $\tau$, as defined above. In terms of this set of variables, the Lagrangian is

$$
\begin{aligned}
L= & \sum_{\alpha=1}^{N}\left[\frac{M}{2} s^{2}\left(L_{x}^{2} \stackrel{*}{\rho}_{x \alpha}^{2}+A \stackrel{*}{\rho_{y \alpha}^{2}}+A \stackrel{*}{\rho_{z \alpha}^{2}}\right)\right. \\
& \left.+\sum_{i=1}^{n} \frac{m_{i}}{2} s^{2}\left(\stackrel{*}{\xi}_{x i \alpha}^{2}+\stackrel{*}{\xi}_{y i \alpha}^{2}+\stackrel{*}{\xi}_{z i a}^{2}\right)\right]-\Phi+\frac{w_{s}}{2} \stackrel{*}{s}^{2} \\
& -(f+1) k_{B} T_{\text {ext }} \ln s+\frac{w_{A}}{2} \stackrel{*}{A}^{2}-P_{\text {ext }} L_{x} A,
\end{aligned}
$$

with constraints,

$$
\begin{aligned}
& \left(\xi_{i a}-\xi_{j a}\right)^{2}-d^{2}=0, \\
& \frac{-1}{M} \sum_{i=1}^{n} m_{i} \xi_{i a}=0,
\end{aligned}
$$

where $N$ is the total number of molecules in the system, $n$ is the total number of atoms in each molecule (in order to keep the notation as simple as possible, we assume that all molecules have the same number of atoms and are indistinguishable), $\mathbf{h}$ is the metric tensor defined in Eq. (3.1), $d$ is the fixed length of the covalent bonds (which we also assume to be constant for simplicity), $M$ is the total mass of the polymer chain, and * indicate time derivatives with respect to the "virtual" time $\tau$. The corresponding Hamiltonian of the " "extended" system is

$$
\begin{aligned}
H= & \sum_{\alpha=1}^{N}\left[\frac{1}{2 M s^{2}}\left(\frac{\Pi_{x \alpha}^{2}}{L_{x}^{2}}+\frac{\Pi_{y \alpha}^{2}}{A}+\frac{\Pi_{z \alpha}^{2}}{A}\right)\right. \\
& \left.+\sum_{i=1}^{n} \frac{1}{2 m_{i} s^{2}}\left(\pi_{x i \alpha}^{2}+\pi_{y i \alpha}^{2}+\pi_{z i \alpha}^{2}\right)\right]+\Phi+\frac{\pi_{s}^{2}}{2 w_{s}} \\
& +(f+1) k_{B} T_{\mathrm{ext}} \ln s+\frac{\pi_{A}^{2}}{2 w_{A}}+P_{\mathrm{ext}} L_{x} A
\end{aligned}
$$


where the momenta of the "extended" variables are now

$$
\begin{aligned}
& \pi_{i \alpha}=m_{i} s^{2} \stackrel{*}{\xi}_{i \alpha}, \\
& \Pi_{x \alpha}=M s^{2} L_{x}^{2} \stackrel{*}{\rho}_{x \alpha}, \\
& \Pi_{y \alpha}=M s^{2} A \stackrel{*}{\rho_{y \alpha},} \\
& \Pi_{z \alpha}=M s^{2} A \stackrel{*}{\rho_{z \alpha}}, \\
& \pi_{s}=w_{s} s, \\
& \pi_{A}=W_{A} \stackrel{*}{A} .
\end{aligned}
$$

The equations of motion using these center of mass "extended" variables are

$$
\begin{aligned}
& m_{i \alpha} s^{2} \stackrel{* *}{\xi}_{i \alpha}=\mathbf{f}_{i \alpha}+\mathbf{g}_{i \alpha}-m_{i \alpha} \mu_{\alpha}-2 m_{i \alpha} \stackrel{*}{s} s \stackrel{*}{\xi}_{i \alpha}, \\
& M s^{2} \stackrel{* *}{\rho}_{x \alpha}=\frac{1}{L_{x}} F_{x \alpha}-2 M \stackrel{*}{s} s \stackrel{*}{\rho}_{x \alpha}, \\
& M s^{2}{\stackrel{* *}{\rho_{y \alpha}}}_{y}=\frac{1}{\sqrt{A}} F_{y \alpha}-2 M \stackrel{*}{s} s{\stackrel{*}{\rho_{y \alpha}}}-\frac{M s^{2} \stackrel{*}{A}}{A} \stackrel{*}{\rho}_{y \alpha}, \\
& M s^{2} \stackrel{* *}{\rho_{z \alpha}}=\frac{1}{\sqrt{A}} F_{z \alpha}-2 M \stackrel{*}{s} s{\stackrel{*}{\rho_{z \alpha}}}-\frac{M s^{2} \stackrel{*}{A}}{A} \stackrel{*}{\rho_{z \alpha}}, \\
& w_{s} \stackrel{* *}{s}=\sum_{\alpha=1}^{N}\left[\left(\sum_{i=1}^{n} m_{i} s \stackrel{*}{\xi_{i \alpha}^{2}}\right)+M s\left(L_{x}^{2} \stackrel{*}{\rho}_{x \alpha}^{2}+A \stackrel{*}{\rho_{y \alpha}^{2}}+A \stackrel{*}{\rho_{z \alpha}^{2}}\right)\right] \\
& -\frac{(f+1) k_{B} T_{\mathrm{ext}}}{s} \\
& w_{A} A * \sum_{\alpha=1}^{N *}\left[\frac{M s^{2}}{2}\left(\stackrel{*}{\rho}_{y \alpha}^{2}+\stackrel{*}{\rho}_{z \alpha}^{2}\right)\right. \\
& \left.+\frac{1}{2 \sqrt{A}}\left(F_{y \alpha} \rho_{y \alpha}+F_{z \alpha} \rho_{z \alpha}\right)\right]-P_{\mathrm{ext}} L_{x},
\end{aligned}
$$

where $\mathbf{f}_{i \alpha}$ is the force on the $i$ th atom of $\alpha$ th molecule which is derived from the interatomic potential, $\mathbf{F}_{\alpha}$ is the total force on molecule $\alpha$. An artificial (constraint) force is added in order to ensure that the bond lengths remain fixed. The sum of the constraint force for the two bonds to atom $i$ of molecule $\alpha$ is $\mathbf{g}_{i \alpha}$. As discussed in Ref. 8, $\mu_{\alpha}=\mathbf{F}_{\alpha} / M$.

The relationships between the extended variables in the center of mass system (Greek letters) and the real variables (Roman letters) are

$$
\begin{aligned}
& \mathbf{R}_{\alpha}=\mathbf{h} \rho_{\alpha}, \\
& \mathbf{r}_{i \alpha}=\mathbf{R}_{\alpha}+\xi_{i \alpha}, \\
& d t=d \tau / s, \\
& P_{x \alpha}=\Pi_{x \alpha} / L_{x} s, \\
& P_{y \alpha}=\Pi_{y \alpha} / \sqrt{A} s,
\end{aligned}
$$

$$
\begin{aligned}
& P_{z \alpha}=\Pi_{z \alpha} / \sqrt{A} s, \\
& \mathbf{P}_{i \alpha}=\mathbf{P}_{i a} \frac{m_{i}}{M}+\frac{\pi_{i \alpha}}{s}, \\
& P_{s}=\pi_{s}, \\
& P_{A}=\pi_{A},
\end{aligned}
$$

where upper and lower case letters refer to the center of mass and atom coordinates, respectively, $R$ and $r$ correspond to positions, and $P$ and $p$ correspond to momenta. The resulting equations of motion in the real variables and in real time are found using Eqs. (4.5) and (4.6),

$$
\begin{aligned}
& m_{i} \ddot{r}_{x i \alpha}=f_{x i \alpha}+g_{x i \alpha}-\dot{s} s^{-1} p_{x i \alpha}, \\
& m_{i} \ddot{r}_{y i \alpha}=f_{y i \alpha}+g_{y i \alpha}-\dot{s} s^{-1} p_{y i \alpha}+\frac{m_{i} R_{y \alpha}}{2 A}\left(\ddot{A}-\frac{\dot{A}^{2}}{2 A}\right) \\
& m_{i} \ddot{r}_{z i \alpha}=f_{z i \alpha}+g_{z i \alpha}-\dot{s} s^{-1} p_{z i \alpha}+\frac{m_{i} R_{z \alpha}}{2 A}\left(\ddot{A}-\frac{\dot{A}^{2}}{2 A}\right) \\
& w_{s} \ddot{s}=w_{s} \frac{\dot{s}^{2}}{s}+s\left[\sum_{\alpha=1}^{N} \sum_{i=1}^{n} \frac{p_{x i \alpha}^{2}+p_{y i \alpha}^{2}+p_{z i \alpha}^{2}}{m_{i}}\right. \\
& \left.\quad-(f+1) k_{B} T\right], \\
& w_{A} \ddot{A}=w_{A} \frac{\dot{s} \dot{s}}{s}+s^{2} L_{x}\left[\frac { 1 } { 2 A L _ { x } } \sum _ { \alpha = 1 } ^ { N } \left(\frac{P_{y \alpha}^{2}+P_{z \alpha}^{2}}{M}+F_{y \alpha} R_{y \alpha}\right.\right. \\
& \left.\left.+F_{z \alpha} R_{z \alpha}\right)-P_{\mathrm{ext}}\right],
\end{aligned}
$$

where the forces with two indices indicate center of mass forces, while those with three indicate forces on atoms within a particular polymer chain. Note that the term in the parentheses in the equation of motion of the cross sectional area is the internal stress associated with the center of mass of the molecules (rather than that of the individual atoms). $\mathbf{R}_{\alpha}, \mathbf{P}_{\alpha}$, and $\mathbf{p}_{i \alpha}$ are known functions of $\mathbf{r}_{i \alpha}, \dot{\mathbf{r}}_{i \alpha}, A$, and $\dot{A}$,

$$
\begin{aligned}
& \mathbf{R}_{\alpha}=\frac{1}{M} \sum_{i=1}^{n} m_{i} \mathbf{r}_{i \alpha}, \\
& P_{x \alpha}=M \dot{R}_{x \alpha}, \\
& P_{y \alpha}=M \dot{R}_{y a}-M \frac{\dot{A}}{2 A} R_{y \alpha}, \\
& P_{z \alpha}=M \dot{R}_{z a}-M \frac{\dot{A}}{2 A} R_{z \alpha}, \\
& p_{x i \alpha}=m_{i} \dot{r}_{x i \alpha}, \\
& p_{y i \alpha}=m_{i} \dot{r}_{y i \alpha}-\frac{m_{i} \dot{A}}{2 A} R_{y \alpha}, \\
& p_{z i \alpha}=m_{i} \dot{r}_{z i \alpha}-\frac{m_{i} \dot{A}}{2 A} R_{z \alpha} .
\end{aligned}
$$


Based upon these relationships, the temporal evolution of the real variables described by Eqs. (4.5) and (4.6) can be shown to rigorously correspond to the $N T L_{x} \sigma_{y y} \sigma_{z z}$ ensemble with constrained intrachain bond lengths.

These equations of motion describe the dynamics of the real variables of each polymer chain and the atoms within them, subject to the fixed bond length constraint along the chain and within the $N T L_{x} \sigma_{y y} \sigma_{z z}$ ensemble. Although these equations of motion were derived using the extended variables, it is the real variables that directly enter the MD simulation code. It is important, however, to realize that the relationship between the real variables in the $N T L_{x} \sigma_{y y} \sigma_{z z}$ ensemble is not exactly the same as in the simpler $N V E$ ensemble. For example, the $y$-component of the momentum of an individual atom $i$ cannot be found directly from the time derivative of the position of that atom, but rather from $p_{\text {aiy }}=m_{i} \dot{r}_{\text {aiy }}-\left(m_{i} \dot{A} / 2 A\right) R_{\alpha y}$. Additionally, other properties must be computed directly in terms of the momenta and positions and not in terms of their derivatives (e.g., the kinetic energy should be written as $\mathbf{p}_{i}^{2} / 2 m$ rather than as $\left.m \dot{\mathbf{r}}_{i}^{2} / 2\right)$.

The equations of motion in Eq. (4.7) have a similar structure to those in Eq. (3.7) for an atomic system. Note that variations in the cross sectional area, $A$, only affect the equation of motion of the atoms in the $y$ and $z$-directions by modifying the position of the center of mass of the entire molecule. The equation of motion for the cross sectional area $A$, in turn, is determined by the difference between the average normal stress internal to the system in the $y$ and $z$-directions and the external pressure. The normal internal stresses are

$$
\sigma_{k k}=V^{-1} \sum_{\alpha=1}^{N}\left(\frac{P_{k \alpha}^{2}}{M}+F_{k \alpha} R_{k \alpha}\right),
$$

where the subscript $k$ refers to the Cartesian directions $x, y$, and $z$. These stresses are "molecular" stresses, because Eq. (4.9) is written directly in terms of the momenta, forces, and coordinates of the centers of mass of the molecules, rather than the atoms.

At constant strain (i.e., fixed $L_{x}$ ) the Hamiltonian of the system should be conserved. In terms of the real variables, the Hamiltonian of the system can be written as

$$
\begin{aligned}
H= & \sum_{\alpha=1}^{N} \sum_{i=1}^{n}\left(\frac{p_{x i \alpha}^{2}+p_{y i \alpha}^{2}+p_{z i \alpha}^{2}}{2 m_{i}}\right)+\Phi \\
& +\frac{p_{s}^{2}}{2 w_{s}}+(f+1) k_{B} T_{\text {ext }} \ln s+\frac{p_{A}^{2}}{2 w_{A}}+p_{\text {ext }} L_{x} A .
\end{aligned}
$$

However, if the system is strained at any finite rate, the Hamiltonian will not be conserved. As in the atomic systems, the molecular system can be strained at a fixed strain rate by quasistatically changing $L_{x}$ according to $d L_{x}=\dot{\epsilon} L_{x} d t$, where $d t$ is the time step used in the MD simulation. In the present molecular system, we translate the centers of mass of the molecules at a rate which is in accordance with the rate at which the macroscopic shape of the simulation cell changes.
This is consistent with our definition of the Lagrangian above. The momenta of atoms in $x$ direction will feel this rate and change to

$$
\begin{aligned}
& p_{x i \alpha}=m_{i} \dot{r}_{x i \alpha}-\frac{m_{i} \dot{L}_{x}}{L_{x}} R_{x \alpha}, \\
& p_{y i \alpha}=m_{i} \dot{r}_{y i \alpha}-\frac{m_{i} \dot{A}}{2 A} R_{y \alpha}, \\
& p_{z i \alpha}=m_{i} \dot{r}_{z i \alpha}-\frac{m_{i} \dot{A}}{2 A} R_{z \alpha} .
\end{aligned}
$$

\section{NUMERICAL METHODS}

In this section, we describe the molecular dynamics algorithm used to integrate the equations of motion [Eq. (4.7)] for all of the particles in the $N T L_{x} \sigma_{y y} \sigma_{z z}$ ensemble with fixed intramolecular bond length constraints. In traditional MD simulation, algorithms such as the Verlet algorithm ${ }^{12}$ are used to integrate these equations of motion. The Verlet algorithm is a direct integration of the second-order equations of motion and is based on current positions, current accelerations and positions from the previous time step. However, there are several features of the present, bond length constrained $N T L_{x} \sigma_{y y} \sigma_{z z}$ ensemble that makes these methods inapplicable in their traditional forms.

The first difficulty is associated with the temperature control. In order to keep the sample isothermal, particle accelerations depend not only on the force but also directly on the particle momenta (i.e., particle velocities). Therefore, the Verlet algorithm cannot be directly applied because current velocities are not available. A variant of the Verlet algorithm, known as the velocity Verlet method ${ }^{13}$ is based upon the same type of integration of the equations of motion, but uses the current velocities instead of the positions from the previous time step. Since, in the present case, we need the current particle velocities, a method based upon the velocity Verlet algorithm should be more appropriate.

In the $N T L_{x} \sigma_{y y} \sigma_{z z}$ ensemble, the temperature and normal stress controls require that the equations for motion of the particles depend on the current first and second derivatives of $A$ and $s$. The second derivatives of $A$ and $s$ depend, in turn, on the current particle momenta. This interdependence problem prevents the direct application of the velocity Verlet method. Fox and Andersen ${ }^{14}$ suggested a procedure to modify the velocity Verlet algorithm to handle this difficulty for atomic systems without adversely affecting the accumulated errors. Their approach is to use the particle momenta from the previous time step to approximate $A$ and $s$ and their derivatives and use these approximate values for integrating the equations of motion of the particles. These new coordinates are then used to make a better approximation to the current values of $A$ and $s$, to be used in the following time step. We will apply these same concepts to the integration of the equations of motion here.

While the Fox and Andersen algorithm ${ }^{14}$ solves the problem of incorporation of $A$ and $s$ into the equations of motion for the particles in an atomic system, it does not 
provide a mechanism for incorporating the constraint forces associated with the fixed bond length in a polymer system. Although the SHAKE approach can be used directly with the Verlet algorithm (including the constraint forces), it cannot be used with the velocity Verlet method since the velocity Verlet algorithm requires the determination of the particle acceleration one time step ahead which is not available when using the SHAKE method. Andersen ${ }^{15}$ overcame this difficulty with his RATTLE scheme for including bond length constraints in a velocity Verlet formulation. This is done by performing two types of iterations for each MD time step; one for updating the positions and one for updating the velocities. In a recent paper, Palmer ${ }^{16}$ suggested a new, single iteration approach within the framework of a velocity Verlet algorithm with bond length constraints in an NVE ensemble. In Palmer's approach, the velocity Verlet algorithm is recast in a form in which the bond length constraint is applied two time steps beyond the current one in order to calculate the constraint force one time step ahead,

$$
\begin{aligned}
& x(t+d t)=x(t)+(d t) \dot{x}(t)+\frac{1}{2}(d t)^{2} \ddot{x}(t) \\
& \dot{x}\left(t+\frac{1}{2} d t\right)=\dot{x}(t)+\frac{1}{2}(d t) \ddot{x}(t) \\
& \dot{x}(t+d t)=\dot{x}\left(t+\frac{1}{2} d t\right)+\frac{1}{2}(d t) \ddot{x}(t+d t) \\
& x(t+2 d t)=x(t+d t)+(d t) \dot{x}(t+d t)+\frac{1}{2}(d t)^{2} \ddot{x}(t+d t) .
\end{aligned}
$$

For each time step, this method requires the current positions, current velocities, and current accelerations (including the constraint force contribution).

We employ this modified velocity Verlet approach in our bond length constrained, $N T L_{x} \sigma_{y y} \sigma_{z z}$ ensemble MD simulations. The equations of motion [Eq. (4.7)] are integrated in the following manner:

(1) We assume that $\mathbf{r}(t), \dot{\mathbf{r}}(t), \ddot{\mathbf{r}}(t), s(t), \dot{s}(t), \ddot{s}(t), A(t)$, $\dot{A}(t)$, and $\ddot{A}(t)$ are all known. From this data, the kinetic energy and internal normal stresses can be determined at the current time $t$. Using these quantities in Eq. (5.1a) allows us to determine the values of all of the variables at the next time step, $\mathbf{r}(t+d t), s(t+d t)$, and $A(t+d t)$.

(2) The values of the velocities (i.e., time derivatives) of all of the variables are determined at the next half-time step from Eq. (5.1b), $\dot{\mathbf{r}}\left(t+\frac{1}{2} d t\right), \dot{s}\left(t+\frac{1}{2} d t\right)$, and $\dot{A}\left(t+\frac{1}{2} d t\right)$.

(3) Next, we approximate the velocities and acceleration of $A$ and $s$ at time $(t+d t)$ in terms of the available information.

We obtain $\ddot{s}(t+d t)$ by using the equation of motion for $s$ in Eq. (4.7) in which the derivative of $s$ on the righthand side of the equation is evaluated at $\left(t+\frac{1}{2} d t\right)$ and the momenta of particles at $t$. Since we are using $\dot{s}\left(t+\frac{1}{2} d t\right)$ and the momenta of particles at $t$ to estimate $\ddot{s}(t+d t)$, the resultant $\ddot{s}(t+d t)$ is approximate, as indicated by a "app': $\ddot{s}_{\text {app }}(t+d t)$. Using $\ddot{s}_{\text {app }}(t+d t)$, $\dot{s}\left(t+\frac{1}{2} d t\right)$ and Eq. (5.1c), we determine an approximate value of $\dot{s}(t+d t)$, which we refer to as $\dot{s}_{\text {app }}(t+d t)$.

We then use $\dot{s}_{\text {app }}(t+d t)$ and the momenta of particles at $t$ (instead of $t+d t$ ) to determine approximate values of $\ddot{A}(t+d t) \quad\left[\right.$ i.e., $\left.\quad \ddot{A}_{\text {app }}(t+d t)\right]$. Using $\quad \ddot{A}_{\text {app }}(t+d t)$, $\dot{A}\left(t+\frac{1}{2} d t\right)$, and Eq. (5.1c), we can approximate $\dot{A}(t+d t)$ [i.e., $\left.\dot{A}_{\text {app }}(t+d t)\right]$.

(4) Determine the total force on each particle $\mathbf{f}(t+d t)$ from the interatomic potentials (including nonbonded and bonded contributions) using the particle coordinates $\mathbf{r}(t+d t)$.

(5) We now determine $\dot{\mathbf{r}}(t+d t), \ddot{\mathbf{r}}(t+d t)$, and $\mathbf{r}(t+2 d t)$ using the approximate values obtained in step (3).

The acceleration in the $x$-direction, may be determined from

$$
\begin{aligned}
\ddot{r}_{x i \alpha}(t+d t)= & \frac{1}{m_{i}}\left[f_{x i \alpha}(t+d t)+g_{x i \alpha}(t+d t)\right. \\
& \left.-\frac{\dot{s}(t+d t)}{s(t+d t)} p_{x i \alpha}(t+d t)\right] \\
= & \frac{1}{m_{i}}\left[f_{x i \alpha}(t+d t)+g_{x i \alpha}(t+d t)\right] \\
& +\frac{\dot{s}(t+d t)}{s(t+d t)} \dot{\epsilon} R_{x \alpha}-\frac{\dot{s}(t+d t)}{s(t+d t)} \dot{r}_{x i \alpha}(t+d t) .
\end{aligned}
$$

While Eq. (5.2) only describes the acceleration in the $x$-direction, similar equations apply in the other two, orthogonal directions. For simplicity, we will only explicitly show the procedure for integrating the equations of motion in the $x$-direction.

Examination of Eqs. (5.2) and (5.1c) shows that $\dot{\mathbf{r}}(t+d t)$ and $\ddot{\mathbf{r}}(t+d t)$ are interdependent and functions of $\mathbf{g}(t+d t)$. These equations can be solved to yield $\dot{\mathbf{r}}(t+d t)$ and $\ddot{\mathbf{r}}(t+d t)$ in terms of $\mathbf{g}(t+d t)$ and other known quantities,

$$
\begin{aligned}
\dot{r}_{x i \alpha}(t+d t)= & \dot{r}_{x i \alpha}\left(t+\frac{1}{2} d t\right)+\frac{d t}{2 m_{i}} f_{x i \alpha}(t+d t) \\
& \left.+\frac{(d t) \dot{s}(t+d t)}{2 s(t+d t)} \dot{\epsilon} R_{x \alpha}+\frac{d t}{2 m_{i}} g_{x i \alpha}(t+d t)\right] \\
& \times\left[1+\frac{(d t) \dot{s}(t+d t)}{2 s(t+d t)}\right]^{-1} \\
= & \dot{r}_{x i \alpha}^{\prime \prime}(t+d t)+(1 / d t) C^{g} g_{x i \alpha}(t+d t), \\
\ddot{r}_{x i \alpha}(t+d t)= & \frac{f_{x i \alpha}(t+d t)}{m_{i}}+\frac{\dot{s}(t+d t)}{s(t+d t)}\left[\dot{\epsilon} R_{x \alpha}-\dot{r}_{x i \alpha}^{\prime \prime}(t+d t)\right] \\
& +\frac{2}{(d t)^{2}} C^{g} g_{x i \alpha}(t+d t) \\
= & \ddot{r}_{x i \alpha}^{\prime \prime}(t+d t)+\frac{2}{(d t)^{2}} C^{g} g_{x i \alpha}(t+d t)
\end{aligned}
$$


where $\ddot{r}_{x i \alpha}^{\prime \prime}(t+d t)$ and $\ddot{r}_{x i \alpha}^{\prime \prime}(t+d t)$ represent the constraint force $(g)$ independent parts of the particle velocity and acceleration and along with $C^{g}$ are defined within Eqs. (5.3) and (5.4). Substituting Eqs. (5.3) and (5.4) into Eq. (5.1d), yields

$$
\begin{aligned}
r_{x i \alpha}(t+2 d t)= & r_{x i \alpha}(t+d t)+(d t) \dot{r}_{x i \alpha}^{\prime \prime}(t+d t) \\
& +\frac{1}{2}(d t)^{2} \ddot{r}_{x i \alpha}^{\prime \prime}(t+d t)+2 C^{g} g_{x i \alpha}(t+d t) \\
= & r_{x i \alpha}^{\prime \prime}(t+d t)+2 C^{g} g_{x i \alpha}(t+d t),
\end{aligned}
$$

where $r_{x i \alpha}^{\prime \prime}$ is defined within the equation. Finally, applying the bond length constraint from the SHAKE algorithm, we obtain $C^{g} g_{x i \alpha}(t+d t)$ from Eqs. (5.3), (5.4), and (5.5). Substituting the resultant $C^{g} g_{x i \alpha}(t+d t)$ back into these equations yield $r_{x i \alpha}(t+2 d t), \dot{r}_{x i \alpha}(t+d t)$, and $\ddot{r}_{x i \alpha}(t+d t)$.

(6) Using the values of $\mathbf{r}(t+d t), \dot{\mathbf{r}}(t+d t)$, and $\ddot{\mathbf{r}}(t+d t)$ from step (5), we can obtain more accurate values of the variables $\dot{s}(t+d t), \ddot{s}(t+d t), \dot{A}(t+d t)$, and $\ddot{A}(t+d t)$. These accurate values of $\dot{s}(t+d t), \ddot{s}(t+d t), \dot{A}(t+d t)$, and $\ddot{A}(t+d t)$ are then used in step (5) to yield accurate values of $r_{x i \alpha}(t+2 d t), \dot{r}_{x i \alpha}(t+d t)$, and $\ddot{r}_{x i \alpha}(t+d t)$.

(7) Next, we increment the MD time by $d t$ and return to step (1).

\section{APPLICATION TO THE DEFORMATION OF POLYETHYLENE}

To demonstrate the applicability of the MD method described above to the deformation of polymer systems, we present a simple application of this method to the isothermal, constant strain rate, uniaxial deformation of a model polyethylene (PE) glass. The goal of this application is to demonstrate that the numerical method described in Sec. V works and that all of the variables are controlled in accordance with the proposed statistical mechanics ensemble.

The amorphous PE employed in this simulation is described in terms of the united atom model, in which the $\mathrm{CH}_{2}$ group is considered as one united or quasiatom. The united atom approximation is used in order to make the study of a relatively large system possible without placing undue demands on computational resources. The united atoms are connected by bonds which are constrained to a fixed length $(0.153 \mathrm{~nm})$. Nonbonded, modified Lennard-Jones (LJ) potential, bond bending and torsional potentials are used to describe the remaining interactions. We employ exactly the same forms of the potentials and parameters as did Brown and Clarke, ${ }^{4}$ with the exception that we modified the Lennard-Jones potential in order to insure that the potential and its first derivative has a smooth cutoff at $2.5 r_{0}$, where $r_{0}$ is the Lennard-Jones length parameter (described below).

The modified Lennard-Jones potential acts on all pairs of united atoms, except those neighboring pairs separated by less than four bonds on one chain. The modification to the Lennard-Jones potential used here (in order to insure a smooth cutoff) was introduced by Broughton and Gilmer, ${ }^{17}$

$$
\Phi_{\mathrm{LJ}}(r)=\left\{\begin{array}{c}
4 \epsilon\left[\left(\frac{r_{0}}{r}\right)^{12}-\left(\frac{r_{0}}{r}\right)^{6}\right]+B_{1} \quad \text { when } \frac{r}{r_{0}} \leqslant 2.3 \\
B_{2}\left(\frac{r_{0}}{r}\right)^{12}+B_{3}\left(\frac{r_{0}}{r}\right)^{6}+B_{4}\left(\frac{r}{r_{0}}\right)^{2}+B_{5} \\
\text { when } 2.3<\frac{r}{r_{0}} \leqslant 2.5 \\
0 \quad \text { when } 2.5<\frac{r}{r_{0}},
\end{array}\right.
$$

where $r$ is the distance between pairs of united atoms, $\epsilon$ $=57 \mathrm{~K} \cdot k_{B}$ is the well-depth of the LJ potential, $n$ $=0.428 \mathrm{~nm}$ is the LJ length parameter and $k_{B}$ is Boltzmann's constant. The constants $B_{1}=0.016132 \epsilon, B_{2}=3136.6 \epsilon, B_{3}$ $=-68.069 \epsilon, B_{4}=-0.083312 \epsilon$, and $B_{5}=0.74689 \epsilon$ are chosen to insure a smooth cut at $r=2.5 r_{0}$. The bending potential is a potential written in terms of the valence angles $\theta$ (defined as the angle between two contiguous covalent bonds),

$$
\Phi_{\text {bending }}(\theta)=\frac{1}{2} k_{\theta}\left(\cos \theta-\cos \theta_{0}\right)^{2},
$$

where $k_{\theta}=520 \mathrm{~kJ} / \mathrm{mol}$. The torsional potential is a fourbody potential written in terms of the dihedral angle $\phi$ (defined by three successive bond vectors),

$$
\Phi_{\text {torsion }}(\phi)=C_{0}+C_{1} \cos \phi+C_{2} \cos ^{2} \phi+C_{3} \cos ^{3} \phi,
$$

where $\quad C_{0}=8.832 \mathrm{~kJ} / \mathrm{mol}, \quad C_{1}=18.087 \mathrm{~kJ} / \mathrm{mol}, \quad C_{2}$ $=4.880 \mathrm{~kJ} / \mathrm{mol}$, and $C_{3}=-31.800 \mathrm{~kJ} / \mathrm{mol}$.

In order to keep the system size small yet without introducing significant surface effects, we employ periodic boundary conditions in all three orthogonal directions. The system simulated here consists of 1500 united atoms arranged into five equal length polymer chains. The method by which the initial amorphous polymer structure was constructed will be presented in a future paper. The simulation results presented below were for runs performed at $100 \mathrm{~K}$, which is below the glass transition temperature of this model amorphous PE. The normal stress in the $y$ and $z$ directions was fixed at atmospheric pressure which, for all practical purposes, is indistiguishable from zero.

The conservation of the Hamiltonian $H$ in Eq. (4.10) for constant $L_{x}$ serves as a sensitive test of the errors associated with a MD algorithm. However, $H$ is not strictly conserved in any MD simulation because of numerical errors. For the sake of computational efficiency, the time step should be as large as possible such that the simulation remains stable. Fluctuations in $H$ of order $0.01 \%$ are generally acceptable. Larger fluctuations, associated with time steps that are too large, tend to destabilize the entire simulation. In the present simulation, we typically employ a time step of $d t=\tau$ $=2.63 \times 10^{-15} \mathrm{~s}$ (i.e., $2.63 \mathrm{fs}$ ). Using this time step, we find fluctuations in $H$ of approximately $0.005 \%$. The size of the fluctuation increases with increasing time step. Andersen ${ }^{18}$ suggested that the rms fluctuation of $H$ should be proportional to $(d t)^{2}$ in Verlet (and equivalent) algorithms. 


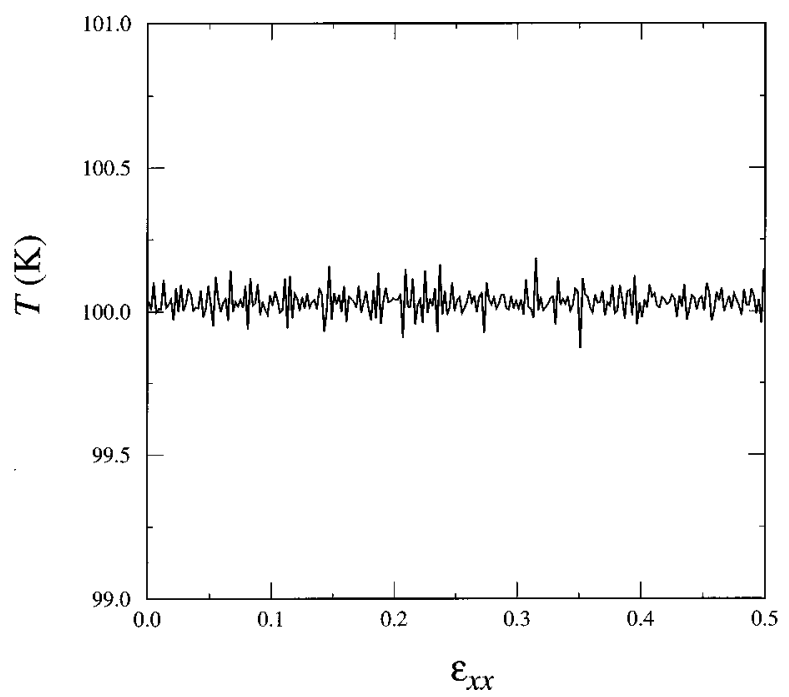

FIG. 2. The variation of the temperature with strain during a constant strain rate $\left(10^{-6} \tau^{-1}\right)$ tensile test. The external temperature $T_{\text {ext }}$ was set at $100 \mathrm{~K}$. All of the data were averaged over 2000 time steps.

For the range of time steps examined for this simulation method $0.5 \tau \leqslant d t \leqslant 2 \tau$ we found that the rms fluctuations in $H$ are indeed proportional to $(d t)^{2}$, which suggests that our algorithm is numerically equivalent to the Verlet algorithm.

In order to simulate a constant strain rate uniaxial deformation test, one dimension of the simulation cell $L_{x}$ was increased at a fixed rate. In the simulations discussed below, the strain rate was fixed at $10^{-6} / \tau$, which means that the length of the sample $L_{x}$ is doubled in $10^{6}$ MD time steps (i.e., an engineering strain of $100 \%$ ). In these simulations, we set $w_{s}=2.0 \times 10^{3}$ amu $\AA^{2}$ and $w_{A}=1.5 \mathrm{amu} \AA^{-2}$. While there is no physical basis to guide the choice of these parameters, we chose these values such that the system equilibrates relatively quickly and remains stable.

Figure 2 shows the variation of the temperature of the system during a constant (engineering) strain rate test. The data in this figure are averaged over 200dt. While the temperature of the system does fluctuate, the average temperature is very nearly constant. The averaged value of temperature over the deformation range in the plot is $100.03 \mathrm{~K}$, which is very close to the prescribed value $100 \mathrm{~K}$. This demonstrates that the temperature control aspect of the algorithm is working properly. The magnitude of the temperature fluctuation is dictated by our choice of the thermal inertia $w_{s}$ and by the degree of averaging used. In addition to controlling the temperature, the algorithm is designed to maintain the normal stresses in the direction perpendicular to the strain axis constant. Figure 3 shows the variation of the average transverse stress $\left(\sigma_{y y}+\sigma_{z z}\right) / 2$ with the applied strain $\epsilon_{x x}$ during a uniaxial tensile test. As with the temperature, the transverse stress fluctuates about a nearly constant value. Careful examination of the data upon which Fig. 3 is based shows that the averaged value of the transverse stress is -0.9 atm, which is close to the prescribed value of -1 atm

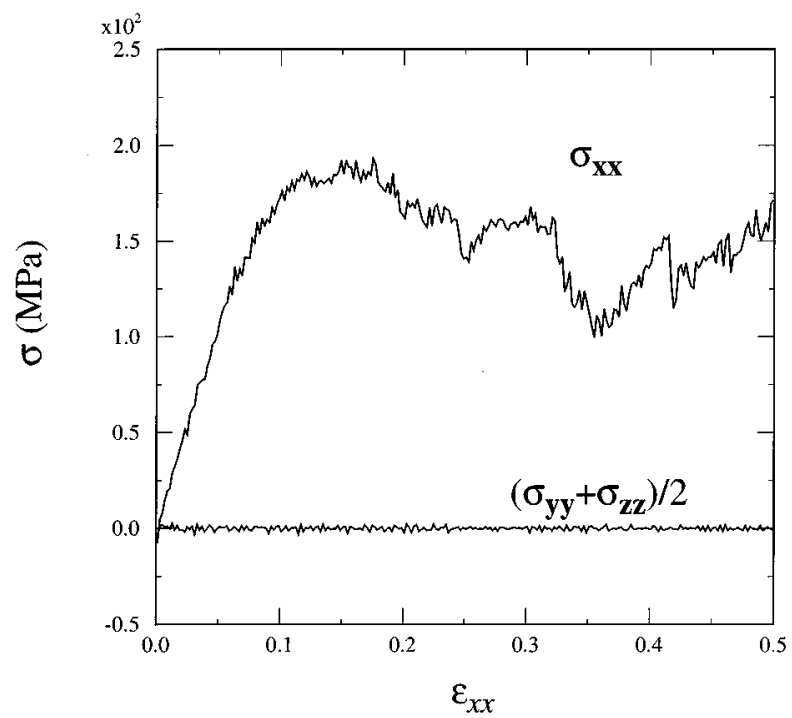

FIG. 3. The tensile $\sigma_{x x}$ and average transverse normal stresses $\left(\sigma_{x x}\right.$ $\left.+\sigma_{y y}\right) / 2$ as a function of strain $\epsilon_{x x}$ in a constant strain rate $\left(10^{-6} \tau^{-1}\right)$ tensile test. All of the data were averaged over 2000 time steps.

$(\approx-0.1 \mathrm{MPa})$. This demonstrates that the transverse stress control aspect of the algorithm is working properly also. The magnitude of the fluctuations (which are large compared with the average) are dictated by our choice of $w_{A}$ and the averaging interval.

In the $x$-direction, the strain is controlled, and we measure the stress. The engineering stress-strain curve associated with the constant strain rate tensile test of our model amorphous polyethylene is presented in Fig. 3. Unlike in a tensile test of a bulk sample, the simulated stress-strain curve is far from smooth. This roughness is real and is not associated with numerical errors. Each small spike in the stress-strain curve is associated with a particular molecular motion/rearrangement. Since the simulation cell from which this data is extracted contains only 1500 united atoms in five macromolecules, each abrupt molecular rearrangement yields a spike in the stress-strain curve. These individual spikes would not be seen in a simulation performed using a very large number of molecules, since the stress is averaged over the entire system. Alternatively, a smooth stress-strain curve could be obtained if we averaged the results over several statistically equivalent simulation runs using a small system.

Several features of the stress-strain curve are notable. The stress-strain curve exhibits a well defined linear elastic regime followed by a stress maximum (ultimate tensile strength) of $0.19 \mathrm{GPa}$ at a strain of approximately $15 \%$. The Young's modulus estimated from the linear region of Fig. 3 is approximately $2.2 \mathrm{GPa}$, which is similar to that reported by Brown and Clarke ${ }^{4}$ for their simulations using a similar potential. The yield stress/strain is subject to our choice of definition. If we define the yield strain in terms of significant deviation from linearity on the scale of the Fig. 3, we find a yield strain of $6 \%$. These high yield and ultimate strengths (and concomitantly high strains) are probably too high for 
bulk, amorphous polyethylene. This is because yield (like fracture) depends on the presence of easy-to-shear regions of the material. As the sample size is increased, more easy-toshear regions and easier-to-shear regions will exist, thereby decreasing the yield/ultimate stress and strain. Additionally, the magnitude of the parameter $w_{A}$ may affect the absolute value of the yield stress/strain as it affects the degree of mechanical constraint. It is important to recall that the strain rates used in the present simulation $\left(\sim 3.8 \times 10^{8} / \mathrm{s}\right)$ are approximately six orders of magnitude larger than the highest strain rates typically used in experiment. Since yielding and flow in amorphous polymers are thermally activated, these high strain rate are expected to have a pronounced effect on the magnitude of the yield and flow stresses. In order to extract realistic yield/flow stresses from simulations of this type, yield/flow stresses could be determined for a range of strain rates and extrapolated into the experimentally accesible regime.

\section{CONCLUSIONS}

In this paper, we have described a new, extended ensemble molecular dynamics algorithm for simulating uniaxial, strain rate controlled tensile tests, the most commonly performed type of mechanical testing. This new algorithm is a generalization of Andersen's ${ }^{9}$ and Nosé' $\mathrm{s}^{11} \mathrm{ex}^{-}$ tended ensemble concept to the constant number of particle $N$, constant temperature $T$, fixed uniaxial displacement $L_{x}$, and fixed transverse stress $\left(\sigma_{y y}\right.$ and $\left.\sigma_{z z}\right)$ ensemble; i.e., the $N T L_{x} \sigma_{y y} \sigma_{z z}$ ensemble. In order to accomplish this goal, we have derived an appropriate Lagrangian, Hamiltonian and equations of motion for all of the degrees of freedom of the system (i.e., the positions and momenta of all particles plus two parameters used to fix the transverse stress and temperature). Since our ultimate application of this new algorithm is the uniaxial deformation of amorphous polymers, we extended this general approach to treat molecular systems in which the intrachain bond lengths are fixed. This is done within the framework of the SHAKE algorithm originally proposed by Ryckaert and co-workers. ${ }^{6-8}$ This was accomplished by rewriting the Lagrangian of the system in terms of the centers of mass of the individual molecules and the relative atomic coordinates. The equations of motion are numerically integrated using a variation of the velocity Verlet algorithm, ${ }^{13}$ modified to account for the bond length constraints. Constant strain rate deformation studies can be performed by increasing the simulation cell size $L_{x}$ at a fixed rate. In order to ensure that this new algorithm corresponds to the $N T L_{x} \sigma_{y y} \sigma_{z z}$ ensemble, we simulated a constant strain rate tensile test of a model amorphous polyethylene system. The results demonstrate that the temperature and transverse stress are well controlled and a reasonable stress-strain curve is obtained. Therefore, we conclude that our new algorithm works and is appropriate for modeling the uniaxial deformation of polymeric systems.

\section{ACKNOWLEDGMENT}

This work was supported by the National Science Foundation (Grant No. DMR 9422049).

\section{APPENDIX}

In this appendix, we demonstrate that the equations of motion derived in Sec. III yield the partition function consistent with the $N T L_{x} \sigma_{y y} \sigma_{z z}$ ensemble. To do this, we follow the approach originally outlined by Nosé. ${ }^{11}$ In an $N T L_{x} \sigma_{y y} \sigma_{z z}$ ensemble, the variation of the energy of the system is described by

$$
d E=T_{\mathrm{ext}} \cdot d s-P_{\mathrm{ext}} \cdot L_{x} \cdot d A .
$$

The partition function associated with Eq. (A1) is

$$
\begin{aligned}
Z_{N T L_{x} P_{y y} P_{z z}}= & \frac{1}{N !} \int A \int d \mathbf{p}^{N} \int d \mathbf{r}^{N} \exp \left\{-\left[E\left(\mathbf{r}^{N}, \mathbf{p}^{N}\right)\right.\right. \\
& \left.\left.+P_{\text {ext }} L_{x} A\right] / k_{B} T_{\text {ext }}\right\},
\end{aligned}
$$

where we use the compact notation $\int d \mathbf{p}^{N}$ $=\int d \mathbf{p}_{1} \int d \mathbf{p}_{2} \cdots \int d \mathbf{p}_{N}$ to indicate multidimensional integrals.

The Hamiltonian associated with the equations of motion derived in Sec. III is given in Eq. (3.4). The partition function associated with the $N T L_{x} \sigma_{y y} \sigma_{z z}$ ensemble is given as the integral over all of the variables (and their momenta), but constrained to satisfy the Hamiltonian (since the Hamiltonian is conserved). Therefore, the partition function of the extended system can be written as

$$
\begin{aligned}
Z= & \frac{1}{N !} \int d \pi_{s} \int d s \int d \pi_{A} \int d A \int d \pi_{x}^{N} \int d x^{N} \\
& \times \int d \pi_{y}^{N} \int d y^{N} \int d \pi_{z}^{N} \int d z^{N} \\
& \times \delta\left[\sum_{i} \frac{1}{2 m_{i} s^{2}}\left(\frac{\pi_{x i}^{2}}{L_{x}^{2}}+\frac{\pi_{y i}^{2}}{A}+\frac{\pi_{z i}^{2}}{A}\right)+\Phi+\frac{\pi_{s}^{2}}{2 w_{s}}\right. \\
& \left.+(f+1) k_{B} T_{\mathrm{ext}} \ln s+\frac{\pi_{A}^{2}}{2 w_{A}}+P_{\mathrm{ext}} L_{x} A-H\right] .
\end{aligned}
$$

Here $\delta(x)$ denotes the Dirac $\delta$ function. By substituting Eqs. (3.2) and (3.8) into Eq. (A3), we can express $Z$ in terms of real variables and their momenta,

$$
\begin{aligned}
Z= & \frac{1}{N !} \int d p_{s} \int d p_{A} \int d A \int d p_{x}^{N} \int d x^{N} \int d p_{y}^{N} \\
& \times \int d y^{N} \int d p_{z}^{N} \int d z^{N} \int d s s^{f} \\
& \times \delta\left[\sum_{i}\left(\frac{p_{x i}^{2}+p_{y i}^{2}+p_{z i}^{2}}{2 m_{i}}\right)+\Phi+\frac{p_{s}^{2}}{2 w_{s}}\right. \\
& +(f+1) k_{B} T_{\mathrm{ext}} \ln s+\frac{p_{A}^{2}}{2 w_{A}}+P_{\mathrm{ext}} L_{x} A-H .
\end{aligned}
$$

Using $\delta[g(s)]=\delta\left(s-s_{0}\right) / g^{\prime}(s)\left[\right.$ where $\left.g\left(s_{0}\right)=0\right]$ and 


$$
E\left(\mathbf{r}^{N}, \mathbf{p}^{N}\right)=\sum_{i}\left[\left(p_{x i}^{2}+p_{y i}^{2}+p_{z i}^{2}\right) / 2 m_{i}\right]+\Phi,
$$

in Eq. (A4), we find

$$
\begin{aligned}
Z= & \frac{1}{N !} \int d p_{s} \int d A \int d p_{A} \int d p_{x}^{N} \int d x^{N} \int d p_{y}^{N} \\
& \times \int d y^{N} \int d p_{z}^{N} \int d z^{N} \int d s \\
& \times \delta\left(s-\exp \left\{-\left[E\left(\mathbf{r}^{N}, \mathbf{p}^{N}\right)+\frac{p_{s}^{2}}{2 w_{s}}+\frac{p_{A}^{2}}{2 w_{A}}\right.\right.\right. \\
& \left.\left.\left.+P_{\mathrm{ext}} L_{x} A-H\right] /(f+1) k_{B} T_{\mathrm{ext}}\right\}\right) \frac{s^{(f+1)}}{(f+1) k_{B} T_{\mathrm{ext}}} \\
= & \frac{1}{(f+1) k_{B} T_{\mathrm{ext}}} \frac{1}{N !} \int d p_{s} \int d A \int d p_{A} \int d p_{x}^{N} \\
& \times \int d x^{N} \int d p_{y}^{N} \int d y^{N} \int d p_{z}^{N} \int d z^{N} \\
& \times \exp \left\{-\left[E\left(\mathbf{r}^{N}, \mathbf{p}^{N}\right)+\frac{p_{s}^{2}}{2 w_{s}}+\frac{p_{A}^{2}}{2 w_{A}}+P_{\mathrm{ext}} L_{x} A-H\right] /\right. \\
& \left.k_{B} T_{\mathrm{ext}}\right\} \cdot
\end{aligned}
$$

Evaluating the integrals in Eq. (A5) as

$$
\begin{aligned}
& \int_{-\infty}^{+\infty} d p_{s} \exp \left(\frac{-p_{s}^{2}}{2 k_{B} T_{\mathrm{ext}} w_{s}}\right)=\left(2 \pi k_{B} T_{\mathrm{ext}} w_{s}\right)^{1 / 2}, \\
& \int_{-\infty}^{+\infty} d p_{A} \exp \left(\frac{-p_{A}^{2}}{2 k_{B} T_{\mathrm{ext}} w_{A}}\right)=\left(2 \pi k_{B} T_{\mathrm{ext}} w_{A}\right)^{1 / 2},
\end{aligned}
$$

yields

$$
Z=\frac{\exp \left(H / k_{B} T_{\text {ext }}\right) 2 \pi\left(w_{s} w_{A}\right)^{1 / 2}}{(f+1)} Z_{N T L_{x} P_{y y} P_{z z}},
$$

where $Z_{N T L_{x} P_{y y} P_{z z}}$ is defined in Eq. (A1). Since the partition function in Eq. (A6) is proportional to the partition functions defined directly in terms of the $N T L_{x} \sigma_{y y} \sigma_{z z}$ ensemble [i.e., Eq. (A1)], we conclude that the equations of motion derived in Sec. III are consistent with the $N T L_{x} \sigma_{y y} \sigma_{z z}$ ensemble. Note: that the coefficient of $Z_{N T L_{x} P_{y y} P_{z z}}$ is a constant and constant proportionality only effect the normalization of the partition function.

\footnotetext{
${ }^{1}$ A. S. Argon, P. H. Mott, and U. W. Suter, Phys. Status Solid B 172, 193 (1992).

${ }^{2}$ P. H. Mott, A. S. Argon, and U. W. Suter, Philos. Mag. 67, 931 (1993).

${ }^{3}$ M. Hutnik, A. S. Argon, and U. W. Suter, Macromolecules 26, 1097 (1993).

${ }^{4}$ D. Brown and J. H. R. Clarke, Macromolecules 24, 2075 (1991).

${ }^{5}$ J. I. McKechnie, R. N. Haward, D. Brown, and J. H. R. Clarke, Macromolecules 26, 198 (1993).

${ }^{6}$ J. P. Ryckaert, G. Ciccotti, and H. J. C. Berendsen, J. Comp. Phys. 23, 327 (1977).

${ }^{7}$ J. P. Ryckaert and G. Ciccotti, J. Chem. Phys. 78, 7368 (1983).

${ }^{8}$ M. Ferrario and J. P. Ryckaert, Mol. Phys. 54, 587 (1985).

${ }^{9}$ H. C. Andersen, J. Chem. Phys. 72, 2384 (1980).

${ }^{10}$ M. Parrinello and A. Rahman, Phys. Rev. Lett. 45, 1196 (1980).

${ }^{11}$ S. Nosé, Mol. Phys. 52, 255 (1984).

${ }^{12}$ L. Verlet, Phys. Rev. 159, 98 (1967).

${ }^{13}$ W. C. Swope, H. C. Andersen, P. H. Berens, and K. R. Wilson, J. Chem. Phys. 76, 637 (1982).

${ }^{14}$ J. R. Fox and H. C. Andersen, J. Phys. Chem. 88, 4019 (1984).

${ }^{15}$ H. C. Andersen, J. Comp. Phys. 52, 24 (1983).

${ }^{16}$ B. J. Palmer, J. Comp. Phys. 104, 472 (1993).

${ }^{17}$ J. Q. Broughton and G. H. Gilmer, J. Comp. Phys. 79, 5095 (1983).

${ }^{18}$ M. P. Allen and D. J. Tildesley, Computer Simulation of Liquids (Clarendon, Oxford, 1987), Chap. 3.
} 